\title{
Les variables socials com a factors de risc, factors de protecció i dificultats consegüents al trastorn del desenvolupament del llenguatge: Una visió holística
}

\section{Mario Valera-Pozo, Daniel Adrover-Roig i Eva Aguilar- Mediavilla}

Grup d'Investigació en Desenvolupament, Educació i Llenguatge (I+DEL), Departament de Pedagogia Aplicada i Psicologia de l'Educació. Universitat de les Illes Balears

eva.aguilar@uib.es

Recepció: 20/04/2020, acceptació: 20/08/2020

Resum: El trastorn del desenvolupament del llenguatge (TDL) es defineix com una dificultat persistent del llenguatge que afecta la vida de les persones que el pateixen. Entre els factors de risc i protecció més freqüents d'aquest trastorn es troben el gènere, la prematuritat $o$ una possible càrrega genètica. No obstant això, existeix també un altre conjunt de factors socials que es relacionen amb el TDL. Aquestes variables socials inclouen elements familiars, socioculturals i econòmics que afecten especialment la qualitat i quantitat de l'estimulació lingüística que rep l'infant, i influeixen en l'adquisició del llenguatge i el seu desenvolupament. En aquest article abordem, des d'una visió holística i integradora, el conjunt d'aquestes variables, el seu funcionament com a factors de risc i de protecció davant el TDL i la seva afectació per la presència del trastorn.

Paraules clau: trastorn del desenvolupament del llenguatge, variables socials, factors de risc i protecció.

Las variables sociales como factores de riesgo, factores de protección y dificultades consecuentes al trastorno del desarrollo del lenguaje: Una visión holística

Resumen: El Trastorno del Desarrollo del Lenguaje (TDL) se define como una dificultad persistente del lenguaje, que afecta a la vida de las personas que lo padecen. Entre los factores de riesgo y protección más frecuentes en este trastorno se encuentran el género, la prematuridad o una posible carga genética. No obstante, existe también otro conjunto de factores sociales que se relacionan con el TDL. Estas variables sociales incluyen elementos familiares, socioculturales y económicos que afectan especialmente a la calidad y cantidad de la estimulación lingüística que recibe el niño, influyendo en la adquisición del lenguaje y en su desarrollo. En este artículo abordamos, desde una visión holística e integradora, el conjunto de estas variables, su funcionamiento como factores de riesgo y de protección ante el TDL y su afectación por la presencia del trastorno. 
Palabras clave: trastorno del desarrollo del lenguaje, variables sociales, factores de riesgo y protección.

Social variables as risk factors, protective factors and difficulties resulting from Developmental Language Disorder: A holistic view

Abstract: Developmental Language Disorder (DLD) can be defined as a persistent language difficulty which affects the lives of individuals who experience it. Among the most frequent risk and protective factors of this disorder, we identify gender, prematurity or a possible genetic influence. However, another group of social variables is also related to DLD. These social variables include familial, sociocultural and economic elements, which especially affect the quality and the amount of linguistic input that children receive, influencing both language acquisition and development. In this article, we address these variables from a holistic and integrative perspective and discuss how they can be considered as risk and protective factors of DLD. The effect they experience from the presence of this disorder is also considered.

Keywords: developmental language disorder, social variables, risk and protective factors.

\section{INTRODUCCIÓ}

El trastorn del desenvolupament del llenguatge (TDL) es caracteritza per la presència de dificultats persistents i severes del llenguatge, que es poden donar tant en la vessant expressiva com en la comprensiva i en qualsevol dels components del llenguatge. Aquest conjunt de problemàtiques arriba al punt d'afectar la vida de les persones que el pateixen, ja que dificulten la seva comunicació, el seu aprenentatge o ambdues coses (Bishop, Snowling, Thompson, Greenhalgh, Whitehouse et al. 2016, Bishop, Snowling, Thompson, Greenhalgh i CATALISE-2 consortium 2017). El TDL sol començar amb un retard del llenguatge i es diagnostica al voltant dels cinc anys, quan les dificultats del llenguatge no han millorat o es preveu que difícilment puguin fer-ho. Prèviament, el TDL era anomenat trastorn específic del llenguatge (Stark i Tallal 1981, Leonard 1998). Per aquest motiu, en aquest treball encabirem ambdós termes, TEL i TDL, com a part del mateix fenomen i sota l'etiqueta de TDL, així com considerarem el retard del llenguatge com un precursor d'aquest trastorn (Collisson, Graham, Preston, Rose, McDonald i Tough 2016).

Tradicionalment, els criteris del TEL incloïen un retard lingüístic d'1,5 desviacions estàndard en alguna prova lingüística estandarditzada, un quocient d'intel-ligència no verbal superior a 70 o 85 (segons els criteris) i absència de dany cerebral, pèrdua auditiva i/o trastorns socioemocionals (Stark i Tallal 1981, Aguado et al. 2015). Aquests criteris indicaven que el retard de llenguatge persistent no podia estar causat per raons mèdiques, biològiques ni orgàniques, així com tampoc per motius cognitius o socials.

Actualment, els criteris per TDL són més oberts i presenten una visió dinàmica (Bishop et al. 2016, Aguilar-Mediavilla, Buil-Legaz, Esteller-Cano i Pérez-Castelló 2019), de manera que les dificultats del llenguatge han d'afectar la vida quotidiana en el moment actual o al llarg del seu desenvolupament, alhora que no hi ha una condició biomèdica associada com la discapacitat intel-lectual, el trastorn de l'espectre autista, la sordesa, trastorns degeneratius o l'afàsia.

Tot i la manca d'una condició biomèdica evident en el TDL, se n'han trobat alguns substrats genètics o familiars (Bishop 2002), així com és cert que hi ha alguns factors protectors o de risc que, si bé no causen l'aparició del trastorn, poden modular-ne la gravetat i el pronòstic a llarg termini (Bishop 2003). D'aquests factors en parlarem en Les variables socials com a factor de risc, factors de protecció i dificultats consegüents al trastorn $\mathbf{1 0 4}$ del desenvolupament del llenguatge: Una visió holística 
aquest article, amb especial atenció a les circumstàncies socials (culturals, econòmiques i familiars) que exerceixen una influència rellevant en aquests casos.

\section{FACTORS DE RISC I FACTORS PROTECTORS}

Entenem per factors de risc del TDL aquelles variables, ja siguin de caire biològic 0 ambiental, que, estadísticament, es relacionen amb el trastorn, tot i que no es pugui establir una causalitat entre aquestes i les dificultats del llenguatge. És a dir, els factors de risc no permeten predir amb solidesa el llenguatge dels infants, però sí que són més freqüents en casos de problemes de llenguatge que en nens i nenes que presenten un desenvolupament típic. A més, cal aclarir que no solen aparèixer de forma aïllada, sinó que se sumen entre ells i es poden presentar conjuntament amb el trastorn, amb la qual cosa augmenta el risc de patir-lo i que les seves conseqüències siguin més greus (Zubrick, Taylor i Christensen 2015). Finalment, cal dir que els factors de risc no són criteris d'exclusió pel TDLi, per tant, encara se'n pot diagnosticar aquest trastorn si hi són presents.

D'altra banda, els factors protectors es poden definir com aquelles característiques o variables que poden ajudar a millorar la salut o el pronòstic d'un trastorn o, almenys, a equilibrar-se amb els factors de risc i impedir que aquest empitjori. De vegades, segons si es configura positivament o negativa, una mateixa variable pot entendre's com a factor de risc o de protecció.

La identificació i valoració dels factors de risc i protectors en els casos de TDL (i, fins i tot, en els de retard de llenguatge) és una eina cabdal de cara a la intervenció que es farà. El motiu rau en el fet que, tot d'una que els professionals pertinents detectin un dèficit en el llenguatge, es recomana una intervenció adient per assegurar un millor pronòstic i, en qualsevol cas, evitar la cronificació de la problemàtica en la mesura que sigui possible. En aquest sentit, tractar no només l'aspecte lingüístic en una intervenció primerenca, sinó també fer prevenció sobre els factors de risc i enfortir els factors protectors, pot afavorir el desenvolupament a llarg termini de l'infant amb retard de llenguatge o sospita de TDL.

\subsection{Factors de risc associats al TDL}

Començant pels factors de risc de TDL, possiblement un dels més coneguts és el de pertànyer al sexe masculí, tot i que encara no disposem de suficients evidències en l'aspecte biològic que ho puguin explicar (Lutchmaya, Baron-Cohen i Raggatt 2002; Font-Jordà, Gamundí, Nicolau-Llobera i Aguilar-Mediavilla 2018). Alguns estudis mostren que els nens poden tenir més probabilitats de patir dificultats de llenguatge, fins el doble que les nenes (Zambrana, Pons, Eadie i Ystrom 2014), tot i que altres estudis amb mostres elevades assenyalen que aquesta ràtio es troba més equilibrada i no hi ha tanta diferència (Tomblin, Records, Buckwalter, Zhang, Smith i O'Brien 1997, Norbury et al. 2016). Tot i això, és cert que aquestes dades depenen del tipus d'avaluació, i les nenes tenen símptomes més subtils que en fan més difícil la identificació, cosa que probablement fa que existeixi un cert infradiagnòstic (Whitehouse 2010). Això és degut, segons Durkin i Conti-Ramsden (2016), a l'associació del gènere masculí amb els problemes de conducta: donat que els nens presenten més freqüentment que les nenes problemes externalitzants, és més fàcil que en ser avaluats per aquest fet es detecti el dèficit lingüístic (si és el cas) durant el procés general d'avaluació. Les nenes, com que no presenten alteracions conductuals tan sovint, passarien més desapercebudes en aquest sentit.

Un altre factor de risc és la prematuritat: els infants que neixen prematurs es troben en més risc de patir problemes de llenguatge (McKean, Wraith, Eadie, Cook, Mensah i Reilly 2017, Bosch, Agut-Quijano i Busquets-Ferrer 2018). A més, aquesta probabilitat va en augment com més prematur hagi estat el nen o la nena, per la qual cosa els infants Les variables socials com a factor de risc, factors de protecció i dificultats consegüents al trastorn 105 del desenvolupament del llenguatge: Una visió holística 
prematurs són casos que cal vigilar, també quan parlem de trastorns de llenguatge. Segons Maggiolo, Varela, Arancibia i Ruiz (2014), el fet que el llenguatge sigui més deficitari en els casos d'infants prematurs es relaciona amb les seqüeles cognitives i relacionades amb la sinaptogènesi, la poda neural i l'arborització dendrítica (fenòmens de caire neuronal) que manifesten molts dels prematurs a mesura que es desenvolupa el seu sistema nerviós central. D'aquesta manera, els autors assenyalen que tant la capacitat discursiva (i la seva coherència) com el processament fonològic i gramatical es veuen afectats per aquestes seqüeles.

Continuant amb aquelles variables que poden incrementar el risc de TDL, en les darreres dècades s'ha posat cert èmfasi a esbrinar la càrrega hereditària i/o genètica de les dificultats del llenguatge. Segons Bishop (2006), el TDL i altres dificultats de llenguatge es poden modular per un factor genètic amb una heretabilitat que pot ser superior al 50\%. Això vol dir que al voltant de la meitat de la variància observada en els dèficits que presenten els casos de TDL es deu a factors genètics. A més, alguns estudis han mostrat que, en el cas de bessons idèntics, és difícil que un d'ells presenti el trastorn i l'altre no (Bishop 2006, Toseeb, Oginni i Dale 2020). Altres estudis troben taxes de TDL més elevades del que s'espera habitualment (generalment 7\%) en algunes poblacions aillades geogràficament (Rakhlin, Kornilov, Palejev, Koposov, Chang i Grigorenko 2013). En aquest darrer cas, la situació d'aïllament afavoreix la transmissió del substrat genètic del trastorn, ja que la presència de diverses famílies amb TDL a la mateixa regió, en la qual els seus fills i filles presenten també les mateixes dificultats lingüístiques en molts de casos, acaba per augmentar la prevalença respecte d'altres zones sense aquesta peculiaritat, degut a l'herència de pares a fills i a la poca variabilitat genètica. Afegit a això, el fet que hi hagi un historial familiar o antecedents de dificultats de llenguatge també sembla augmentar el risc que els infants pateixin un retard de llenguatge de manera persistent, segons reflecteixen alguns estudis (Reilly et al. 2010, Zambrana, Pons, Eadie i Ystrom 2014, Collisson et al. 2016, Ferinu, Ahufinger, Pacheco-Vera, Sanz-Torrent i Andreu 2020), fet que donaria suport a la importància de l'historial familiar com a contribució genètica en aquests casos.

Cal remarcar que aquests factors no causen per si sols el trastorn, sinó que el fan més probable, especialment quan un conjunt d'aquests es donen alhora (Bishop 2001). A més, hi ha altres factors addicionals que poden exercir de factors de risc, però també de factors protectors si es configuren adequadament i que seran objecte de la següent secció.

\subsection{Variables socials en el TDL}

Les variables socials constitueixen un ampli conjunt d'aspectes ambientals que s'han trobat relacionades en diferents estudis amb l'adquisició del llenguatge i el seu desenvolupament, actuant com a factors de risc o protectors. En aquest sentit, es tracta de variables que, quan es manifesten negativament, es troben presents més sovint en els infants amb TDL però amb les quals no s'estableix una relació causal (el fet que existeixi aquest factor no causa el TDL), com explicàvem abans. La seva presència pot també estar lligada no tant al trastorn de llenguatge en si, sinó al risc més elevat de presentar dificultats associades quan es manifesten negativament.

Per variables socials fem referència especialment a elements familiars, socials, culturals i econòmics que afecten sobretot la qualitat i quantitat d'estimulació lingüística que rep l'infant, i que oscil-len des de casos de baixa estimulació a d'altres on directament poden arribar a ocórrer negligències educatives o situacions molt adverses (Sureda-García, Valera-Pozo i Aguilar-Mediavilla 2019). Tot i que els factors ambientals són més propis de l'entorn que de l'infant, són de gran rellevància perquè, tot i no ser la causa del trastorn, marquen la pauta per dissenyar una bona prevenció en casos de trastorns del llenguatge. 
En consonància amb treballs duts a terme amb altres problemàtiques (Shogren, Luckasson i Schalock 2014), aquestes variables de caire contextual es poden classificar com a independents (no manipulables, perquè són de caire personal i/o estable al llarg del temps, o bé rauen fora de l'abast del professional o la societat) o intervinents (modificables amb la intervenció professional o les polítiques socials, degut a la seva qualitat per poder ser millorades i/o al seu dinamisme). Conèixer la naturalesa de cada una ajudarà no només a la prevenció, sinó també a la mateixa intervenció. Podeu consultar la taula 1 per a una enumeració de les variables tractades en aquest article, així com les seves implicacions i naturalesa.

TAULA 1. Resum de variables socials que poden influir en el TDL

$\begin{aligned} & \text { Variables } \\ & \text { Socials }\end{aligned} \quad$ Implicacions $\quad$ Naturalesa $\quad$ Referències

Nivell

socioeconòmic familiar

Suport social i comunitari

Formació musical

Nivell educatiu dels pares

(Aguaded Gómez 2000, Reilly et al. 2010, Hirsh-Pasek et al. 2015, ContiRamsden i Durkin 2016, Gilkerson et al. 2017, Romeo et al. 2018, Auza-

Benavides, Peñaloza i Murata 2019, Ferinu et al, 2020)

Afecta el llenguatge en general

\section{Modula les interaccions rebudes, afecta el llenguatge en general}

Millora el discurs, el vocabulari i la lectura

Intervinent

Fins a cert punt, intervinent 2015, SuredaGarcía et al. 2019)

(Kraus, Hornickel, Strait, Slater i Thompson 2014)

(Maria-Mengel i Linhares 2007, Auza-Benavides et al. 2019)

(Tamis-LeMonda, Song, Leavell, Kahana-Kalman i Yoshikawa 2012, Smith, Levickis, Eadie, Bretherton Conway i Goldfeld 2019) 
Sobreprotecció/ adversitats en la criança

$\begin{array}{cc} & \text { Millora el } \\ & \text { llenguatge en } \\ \text { general i } \\ \text { Implicació } & \text { augmenta el } \\ \text { familiar } & \text { benestar } \\ & \text { emocional i } \\ \text { personal }\end{array}$

Qualitat

d'estimulació

lingüística i

menys varietat

en aquesta

Millora el

enguatge en

benestar

personal
Intervinent

(Aguado 2014,

Smith et al. 2019)

(Collisson et al, 2016, Valera-Pozo, Adrover-Roig,

Intervinent Pérez-Castelló, Sanchez-Azanza i Aguilar-Mediavilla 2020)

Començant per les variables socials, la primera que cal esmentar és el nivell socioeconòmic de la família en la qual creix l'infant. Es tracta d'un factor que pot relacionarse amb l'input lingüístic que rep la persona, així com, al mateix temps, és determinant per altres variables que també el poden afectar com són la residència familiar, la localitat on es troba, el centre educatiu al qual assistirà l'infant i els recursos i tractaments als quals podrà accedir (Sureda-García et al. 2019). Diversos estudis (Aguaded-Gómez 2000, Hirsh-Pasek et al. 2015, Conti-Ramsden i Durkin 2016, Gilkerson et al. 2017, Romeo et al. 2018, AuzaBenavides et al. 2019) han relacionat el fet de créixer en un ambient socioeconòmic empobrit amb una estimulació lingüística més deficitària, que, conjuntament amb altres factors com els que explorem en aquest article, poden relacionar-se amb un desenvolupament del llenguatge per davall del normatiu, que pot arribar a ser compatible amb un diagnòstic de TDL si s'acompleixen els criteris (Reilly et al. 2010, Ferinu et al. 2020). En aquest sentit, sembla que el problema rau realment en el fet que, quan aquest factor és deficitari, la qualitat de l'estimulació lingüística és baixa, i els infants en aquests ambients estan exposats a menys converses, a un lèxic més reduït i a unes interaccions lingüístiques de menys qualitat en comparació amb els seus homònims de famílies en condicions econòmiques mitjanes o altes. Els estudis esmentats indiquen que el component de llenguatge més afectat és el lèxic, per la qual cosa el coneixement i l'ús de vocabulari dels nens i nenes en ambients desfavorits és més pobre i restringit que el de la resta. Aquest fet és lògic, donat que el lèxic serà més ric a com més paraules sigui exposat l'infant. No obstant això, tampoc podem oblidar que alguns estudis (Aguaded-Gómez 2000) assenyalen que el component fonètic-fonològic, especialment pel que fa a l'articulació oral, també està afectat en casos d'ambients empobrits.

Una altra variable de caire social amb influència sobre el rendiment lingüístic de l'infant és el suport que pot rebre per part de la seva família (entenent com a família els membres més enllà de mare, pare i germans), la comunitat del lloc on viu i l'escola a la qual assisteix. Evidentment, aquests altres agents també participen en el desenvolupament del nen o nena, que no només es relaciona amb la seva família més directa. Si la qualitat i la quantitat de l'input que ofereix l'entorn més allà de la família immediata són baixes o inexistents, el llenguatge se'n podrà ressentir i, si és ric, es podrà desenvolupar de manera més favorable (Hirsh-Pasek et al. 2015, Sureda-García et al. 2019).

Quant a la família propera, és a dir, els pares, hi ha altres factors que influeixen en el perfil lingüístic de l'infant. En primer lloc, un dels més estudiats ha estat el nivell educatiu dels progenitors, incorporant o no la categoria laboral de la feina que desenvolupen professionalment. Alguns estudis (Maria-Mengel i Linhares 2007, Auza-Benavides et al. 
2019) afirmen que podria tractar-se d'un factor protector, ja que, com millor categoria professional i nivell d'estudis tinguin els pares (especialment la mare), es poden esperar menys dificultats del llenguatge i un millor desenvolupament de l'infant.

Un altre factor que pot influir és la cultura d'origen dels pares i, més concretament, de la mare; així, les diferències entre cultures en l'ús que es fa del llenguatge poden arribar a ser una variable diferenciadora (Tamis-LeMonda et al. 2012). En aquest sentit, hi ha cultures que empren més la imitació de gestos, sobretot les que no són associades als territoris anglosaxons (Smith et al. 2019), però també n'hi ha d'altres que utilitzen més el llenguatge per referir-se a l'aquí i a l'ara, en comptes dels desitjos o plans de futur (TamisLeMonda et al. 2012). D'aquesta manera, els diversos components del llenguatge, com per exemple la pragmàtica (que també es pot veure afectada en casos d'infants immersos en ambients amb baix nivell socioeconòmic, Tough 1987) o el llenguatge no verbal, es poden veure més o manco potenciats o negligits segons la cultura d'origen.

D'altra banda, Aguado (2014) assenyala també l'estil parental com un factor que pot relacionar-se amb les dificultats lingüístiques de l'infant, especialment un estil caracteritzat per la sobreprotecció. Segons aquest autor, el fet que els pares siguin excessivament protectors es relaciona amb l'aparició de retard del llenguatge i amb el seu manteniment en el temps. Això és degut al fet que els progenitors que tenen aquest estil parental produeixen menys elaboracions lingüístiques complexes dirigides als seus fills o filles, $\mathrm{i}$ tendeixen a simplificar-les, cosa que impedeix que l'infant rebi un input de més qualitat a la llarga. En aquesta relació entre estil parental i problemàtiques del llenguatge també poden influir altres elements. D'aquesta manera, Smith i els seus companys (2019) assenyalen que, en etapes primerenques, els tipus de parentalitat marcats per condicions adverses (mare primerenca, sense suport de la parella o amb problemes de salut mental) s'associen amb un llenguatge menys desenvolupat dels seus fills. No obstant això, cal indicar que aquests problemes no provenen tant de l'adversitat manifestada com dels comportaments parentals que es duen a terme: el llenguatge es veu més afectat quan les directrius verbals cap a l'infant en situació de comunicació no són eficaces i aquest no les respecta. Aquesta situació, per tant, estaria derivada del fet que aquests progenitors tindrien menys recursos comunicatius per adreçar-se als seus fills $i$, en conseqüència, aquests perdrien oportunitats comunicatives, per no atendre-les.

A més, la mateixa implicació familiar, entesa com aquells comportaments, preocupacions i participació en els àmbits de la llar i l'escola per afavorir el desenvolupament de l'infant, també pot arribar a establir-se com un factor protector, donat que sol anar aparellada a l'augment de qualitat de l'estimulació lingüística proveïda a l'infant i, per tant, a un millor pronòstic de trastorn del llenguatge, a més de relacionar-se amb el benestar emocional i amb la salut de l'infant (Sureda-García et al. 2019, Valera-Pozo et al. 2020). El fet que els progenitors, especialment la mare, participin activament en la criança de l'infant (i aquesta no recaigui en altres agents, com per exemple una mainadera o l'estat, en casos d'orfandat) també s'ha mostrat com a factor protector, ja que s'ofereixen situacions comunicatives més riques i variades, en què s'augmenta sobretot el lèxic (Collisson et al. 2016). També la implicació dels progenitors mitjançant la pràctica del joc informal o la lectura diària conjunta pot actuar com a factor protector en casos de trastorns del llenguatge (Collisson et al. 2016).

Finalment, existeix un altre factor, de caire comunitari, menys estudiat, que pot actuar com a variable protectora en els casos de famílies empobrides o en situacions socialment difícils: la formació musical i l'exposició a la música (Kraus et al. 2014). Kraus et al. (2014) han demostrat que, en aquests casos, les condiciones auditives de l'entorn (nivell de renou, exposició a estímuls perjudicials) poden ser inadequades, i afectar tant la lectura com 
diferents variables cognitivolingüístiques. En aquest sentit, el seu estudi demostrà que la formació en algun instrument musical, proveïda per programes comunitaris i de manera regular i constant, pot millorar les habilitats cognitives i lingüístiques dels infants, especialment les referides a la codificació de la parla en l'àmbit neural, la comprensió del discurs, l'adquisició de vocabulari i la fluència lectora. Per aquest motiu, tant l'exposició a la música com la formació musical poden actuar com a factors protectors davant les dificultats associades al TDL.

Tot i que en aquesta secció tractem diverses variables de caire familiar, ja que la família és un dels entorns més habituals en què es pot desenvolupar el llenguatge de l'infant, cal dir que la relació entre aquestes variables i el llenguatge és just una associació, però no una relació causal, com ja hem esmentat abans. D’aquesta manera, la intenció del professional del llenguatge no ha de ser culpabilitzar les famílies, sinó ajudar-les i donar-los estratègies que puguin comportar un millor pronòstic. No hem d'oblidar, així mateix, que el TDL pot aparèxer també en ambients i configuracions de variables més afavorides, tot i que es pugui associar amb més freqüència amb les circumstàncies esmentades.

\section{CONSEQÜÈNCIES EDUCATIVES I SOCIOEMOCIONALS EN EL} TDL

Les variables de caire social i emocional, a més de funcionar com factors de risc o protectors en el TDL, es poden veure afectades a conseqüència d'aquest trastorn. En aquest sentit, estudis previs (Marton, Abramoff i Rosenzweig 2005, Lindsay, Dockrell i Strand 2007, St Clair, Pickles, Durkin i Conti-Ramsden 2011, Yew i O'Kearney 2013, 2015, Bakopoulou i Dockrell 2016, Özcebe, Noyan, Erbas i Karahan Tiğrak 2019) han assenyalat que les persones amb TDL tenen més dificultats per interactuar en situacions socials, a més de presentar més dificultats conductuals i emocionals que les persones que no tenen el trastorn.

Així doncs, els problemes de conducta, com els problemes externalitzants, són susceptibles d'esdevenir un indicador indirecte del trastorn i poden arribar a ser fins el doble que a la població normativa, taxa que també s'aplica als problemes internalitzants (McCabe 2005, Yew i O'Kearney 2013). Els nens i nenes amb TDL manifesten més símptomes conductuals, de tipus ansiós i depressiu (Spaulding, Tammie, Plante i Vance 2008, Van den Bedem et al. 2018), així com són més vulnerables a aquestes circumstàncies. En molts casos, això s'explica per les pròpies dificultats de llenguatge i pels problemes comunicatius que pateixen aquests infants i joves, especialment quan no poden entendre bé la resta de companys ni poden processar adequadament els seus estats i necessitats emocionals (Samson, Van den Bedem, Dukes i Rieffe 2020). Aquesta ansietat o, fins i tot, por a les situacions socials i a l'escrutini dels altres, dels que pensen que els avaluaran negativament, fa que la taxa d'ansietat social sigui fins a tres vegades superior en els casos de TDL respecte a la població normativa, a més d'indicar més patiment per aquest motiu (Voci, Beitchman, Brownlie i Wilson 2006, Wadman, Durkin i Conti-Ramsden 2011).

No obstant això, possiblement el factor que més s'ha estudiat els últims anys és la dificultat emocional que aquesta problemàtica social comporta. Així, els nens i joves amb TDL informen d'índexs d'autoestima més baixos que els seus companys (Lindsay, Dockrell i Mackie 2008, Wadman et al. 2011). Tot i que en l'adultesa millora, l'autoestima, que és factor protector davant les dificultats emocionals, quan és baixa té un impacte negatiu en l'ambient social en forma d'una menor autoconfiança a l'hora de comunicar-se i més por davant del que pensaran els altres. A més, el TDL s'ha demostrat com un factor predictor de futurs problemes en l'aspecte emocional (Spaulding et al. 2008), que s'associen a dificultats per regular les emocions davant situacions adverses, així com per seleccionar les 
estratègies d'actuació adequades, a més de les mateixes dificultats afegides a causa del trastorn per expressar bé les pròpies emocions i entendre les dels altres (Van den Bedem et al. 2018, St Clair, Forrest, Yew i Gibson 2019, Samson et al. 2020).

Relacionat amb els aspectes socials i emocionals que estem abordant, hem de citar la victimització deguda a l'assetjament escolar com un altre aspecte que cal tenir en consideració. D’acord amb alguns estudis, el risc de patir assetjament escolar i la seva mateixa aparició és superior en els infants amb TDL comparat amb altres trastorns o amb la població normotípica (Durkin i Conti-Ramsden 2010, Redmond 2011, Buil-Legaz, Valera-Pozo et al. 2019). No obstant, treballs recents en el nostre entorn (IbáñezRodríguez, Ahufinger, Ferinu, García-Arch, Andreu i Sanz-Torrent 2020) matisen que només la victimització deguda a les dificultats del llenguatge (p. ex. riure's de la manera de parlar) és més alta en els infants amb TDL, però no presenten més victimització de caire general o per altres motius. Considerant això, encara manquen estudis per conèixer exactament quins factors participen en aquesta relació i quines conseqüències exactament pot tenir un fenomen tan rellevant com l'assetjament escolar en una població vulnerable com és la que estem tractant en aquest article.

Tot i el risc de patir aquestes conseqüències socioemocionals en el TDL, hi ha una sèrie de recomanacions que són vàlides per intentar-ne minvar l'efecte en els infants amb TDL. La mateixa implicació familiar pot afavorir el desenvolupament social de l'infant, relacionant-se també amb menys problemes conductuals (El Nokali, Bachman i VotrubaDrzal 2010, Serna i Martínez 2019, Valera-Pozo et al. 2020). Addicionalment, ensenyar els nens i nenes a parlar de les pròpies emocions, així com entrenar la consciència emocional (conèixer quina emoció estan vivint en cada moment i com actuar) i a interpretar les emocions dels altres pot ajudar a minvar els problemes associats al TDL referents al seu desenvolupament socioemocional (Samson et al. 2020). Finalment, estimular les habilitats socials i la conducta prosocial té un efecte positiu en aquests casos, ja que millorar les relacions personals, l'ajust emocional i potenciar les estratègies de resolució de problemes redueix les conductes antisocials $\mathrm{i}$, en definitiva, reforça la resiliència dels nens i nenes amb TDL (Conti-Ramsden i Durkin 2016, Toseeb i St. Clair 2020).

\section{CONCLUSIONS}

Les variables socials tenen un impacte força important en l'adquisició i desenvolupament del llenguatge, a diversos nivells. De fet, aquestes variables es poden retroalimentar entre elles i produir un cercle d'influència mútua. Així, les dificultats socials, econòmiques, familiars i/o culturals poden provocar un input lingüístic de baixa qualitat $\mathrm{i}$ quantitat que pot relacionar-se amb les dificultats del llenguatge. Aquestes dificultats del llenguatge afecten l'adquisició de les habilitats socials i l'autoestima dels infants, que els pot abocar a tenir menys relacions socials, fet que pot comportar dèficits en àmbit social, cultural, familiar i econòmic (vegeu gràfic 1 ).

D'aquesta manera, serà important conèixer aquestes variables i la seva naturalesa (independent o intervinent), per tal de discernir en quines el professional pot actuar-hi de forma més directa, en quines es dependrà dels avenços en polítiques i suports socials i de quines n'haurà de modular l'impacte en el llenguatge en la mesura que sigui possible perquè poden ser difícilment modificables en si mateixes. Fins i tot tenint això en compte, no sempre serà fàcil intervenir-hi, ja que cada família té la seva idiosincràsia i no sempre el professional podrà actuar sobre les circumstàncies que envolten un cas. Tot i aquesta dificultat, serà important treballar sobre les variables socials intervinents per intentar apropar a les famílies i a l'infant el suport i recursos que puguin necessitar, per tal d'ajudarlos, sense culpabilitzar els agents intervinents. S'ha de tenir en compte, a més, que, si bé Les variables socials com a factor de risc, factors de protecció i dificultats consegüents al trastorn $\mathbf{1 1 1}$ del desenvolupament del llenguatge: Una visió holística 
hem tractat aquests aspectes com a factors de risc, molts d'aquests poden esdevenir factors protectors si es configuren correctament o es treballen adequadament.

GRÀFIC 1. Influència mútua entre les variables socials en les dificultats del llenguatge
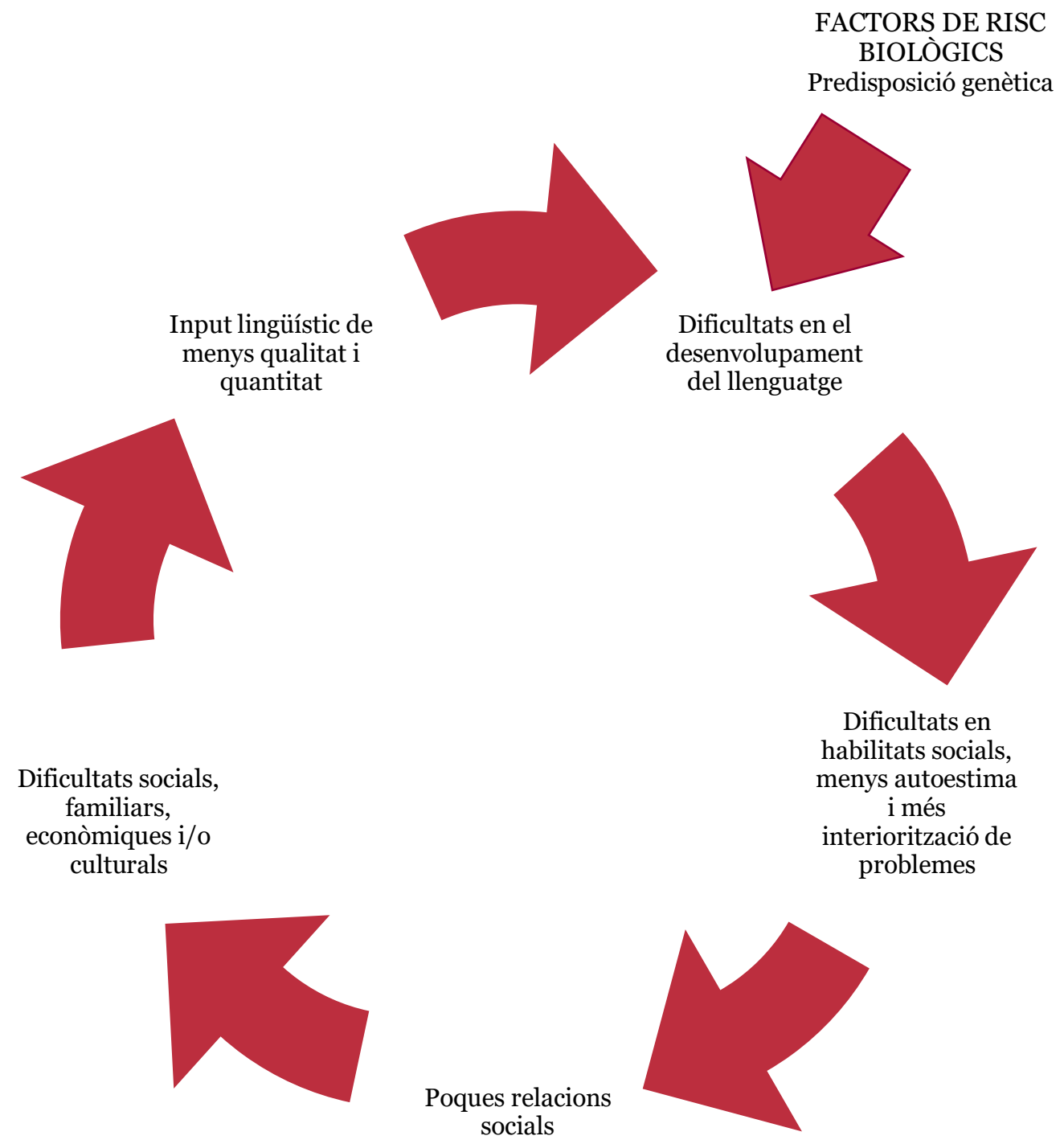

Font: Producció pròpia

Per finalitzar, tot i que existeix un substrat genètic per als trastorns de llenguatge, no podem oblidar que l'entorn pot protegir davant d'aquesta predisposició biològica, fet que demana ampliar la intervenció i tenir en consideració aquests factors socials i ambientals. A més, encara necessitem més investigació sobre els aspectes biològics i els antecedents familiars que es troben darrere el TDL. Per aquest motiu, la intervenció logopèdica i professional serà imprescindible, ja que la mera actuació amb les variables relacionades no és suficient per millorar el pronòstic del trastorn. 


\section{AGRAÏMENTS}

Aquest treball ha estat finançat per FEDER/Ministerio de Economía, Industria y Competitividad _Agencia Estatal de Investigación/EDU2017-85909-P, SEJ2006-12616 and EDU2013-45174-P, i FPU 16/02650.

\section{REFERÈNCIES BIBLIOGRÀFIQUES}

Aguaded Gómez, Ma Cinta (2000). «La participación de la familia en el desarrollo del lenguaje y en el aprendizaje escolar». Revista de Educación XXI, 2(313-318), 313-318.

Aguado, Gerardo (2014). «Retardo del lenguaje». A J. Peña-Casanova (ed.), Manual de Logopedia. Barcelona: Elsevier-Masson.

Aguado, Gerardo; Coloma, Carmen Julia; Martínez, Ana Belén; Mendoza-Lara, Elvira; Montes, Armando; Navarro, Raúl; Serra-Raventós, Miquel (2015). «Documento de consenso elaborado por el comité de expertos en TEL sobre el diagnóstico del trastorno». Revista de Logopedia, Foniatría y Audiología, 35(4), 147-149.

Aguilar-Mediavilla, Eva; Buil-Legaz, Lucía; Esteller-Cano, Àngels; PÉREZ-Castelló, JosEP A. (2019). «Del trastorn específic del llenguatge (TEL) al trastorn del desenvolupament del llenguatge (TDL): un canvi de concepció sobre els trastorns del llenguatge». LSC: Llengua, Societat i Comunicació / Language, Society \& Communication, 17, 64-79.

Auza-Benavides, Alejandra; Peñaloza, Christian; Murata, Chiharu (2019). «The influence of maternal education on the linguistic abilities of monolingual Spanish-speaking children with and without Specific Language Impairment». Atypical Language Development in Romance Languages, 93-112.

Bakopoulou, IoAnNa; Dockrell, Julie E. (2016). «The role of social cognition and prosocial behaviour in relation to the socio-emotional functioning of primary aged children with specific language impairment». Research in Developmental Disabilities, 49-50, 354370 .

Bishop, DOROTHY V. M. (2001). "Genetic influences on language impairment and literacy problems in children: Same or different?». Journal of Child Psychology and Psychiatry, 42, 189-198.

Bishop, DoRothy V. M. (2002). «The role of genes in the etiology of specific language impairment». Journal of Communication Disorders, 35(4), 311-328.

BishoP, DoROTHY V. M. (2003). «Genetic and environmental risks for specific language impairment in children». International Journal of Pediatric Otorhinolaryngology, 67, S143-S157.

Bishop, Dorothy V. M. (2006). "What Causes Specific Language Impairment in Children?». Current directions in psychological science, 15(5), 217-221.

Bishop, Dorothy V. M.; Snowling, Margaret J.; Thompson, Paul A.; Greenhalgh, TRISHA; AdAMs, CATHERINe; CATALISE CONSORTIUM. (2016). "CATALISE: A multinational and multidisciplinary Delphi consensus study. Identifying language impairments in children». PLOS ONE, 11(7), e0158753.

Bishop, Dorothy V. M.; Snowling, Margaret J.; Thompson, Paul A.; Greenhalgh, TRISHA; CATALISE-2 CONSORTIUM (2017). «Phase 2 of CATALISE: A multinational and multidisciplinary Delphi consensus study of problems with language development: Terminology». The Journal of Child Psychology and Psychiatry, 58(10), 1068-1080.

Bosch, Laura; Agut QuiJANo, Thais; Busquets FerRer, Lourdes (2018). «Prematuridad y dificultades del lenguaje». A Aguilar-Mediavilla, Eva; Igualada, Alfonso (ed.),

Les variables socials com a factor de risc, factors de protecció i dificultats consegüents al trastorn $\mathbf{1 1 3}$ del desenvolupament del llenguatge: Una visió holística 
Dificultades del habla, el lenguaje y la comunicación en los trastornos del neurodesarrollo. Barcelona: UOC.

Buil-Legaz, lucía; Valera-Pozo, Mario; Salom-Mariano, Ester; Esteller-Cano, Àngels; Sureda-García, InMaculada; PÉrez-Castelló, Jose A.; LóPEZ-PEnadÉs, RAúl; Sanchez-AZanza, Victor A.; Aguilar-Mediavilla, Eva; Adrover-Roig, Daniel (2019). «Relationship between reported oral language difficulties and the risk of bullying in the primary schooling stage». A XIV International Symposium of Pshycholinguistics (p. 75). Tarragona.

Collisson, Beverly Anne; Graham, Susan A.; Preston, Jonathan L.; Rose, M. Sarah; MCDONALD, SheIla; Tough, SuZANNE (2016). «Risk and protective factors for late talking: An epidemiologic investigation». Journal of Pediatrics, 172, 168-174.e1.

Conti-Ramsden, Gina; Durkin, KEVIn (2016). «What factors influence language impairment? Considering resilience as well as risk». Folia Phoniatrica et Logopaedica, 67(6), 293-299.

Durkin, Kevin; Conti-Ramsden, Gina (2010). «Young people with specific language impairment: A review of social and emotional functioning in adolescence». Child Language Teaching and Therapy, 26(2), 105-121.

El Nokali, Nermeen E.; Bachman, Heather J.; Votruba-Drzal, Elizabeth (2010). «Parent Involvement and Children's Academic and Social Development in Elementary School». Child Development, 81(3), 988-1005.

Ferinu, Laura; Ahufinger, Nadia; Pacheco-Vera, Fernanda; Sanz-Torrent, Mònica; ANDREU, LLORENÇ (2020). "Antecedentes familiares, factores sociodemográficos y dificultades lingüísticas en el trastorno del desarrollo del lenguaje». Revista de Logopedia, Foniatría y Audiología.

Font-Jordà, Antònia; Gamundí, Antoni; Nicolau llobera, María Cristina; AguilarMediavilla, Eva (2018). «Uso del índice digital D2:D4 como indicador biológico del trastorno específico del lenguaje». Anales de Pediatría.

Gilkerson, Jill; Richards, JefFrey A.; Warren, Steven F.; Montgomery, Judith K.; Greenwood, Charles R.; Oller, D. Kimbrough; Hansen, John H. L.; Paul, Terrance D. (2017). «Mapping the Early Language Environment Using All-Day Recordings and Automated Analysis». American Journal of Speech-Language Pathology, 26(2), 248-265.

Hirsh-Pasek, Kathy; Adamson, Lauren B.; Bakeman, Roger; Onen, Margaret Tresch; Golinkoff, Roberta Michink; Pace, Amy; Yust, Paula K. S.; Suma, Katharine (2015). «The Contribution of Early Communication Quality to Low-Income Children's Language Success». Psychological Science, 26(7), 1071-1083.

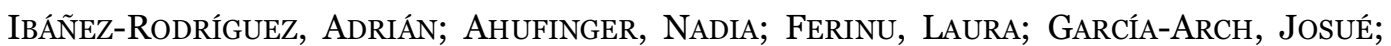
ANDREU, LLORENÇ; SANZ-TORRENT, MònICA (2020). «Dificultades sociales, emocionales y victimización específica por el lenguaje en el trastorno del desarrollo del lenguaje». Revista de Logopedia, Foniatría y Audiología.

Kraus, Nina; Hornickel, Jane; Strait, Dana L.; Slater, Jessica; Thompson, Elaine (2014). «Engagement in community music classes sparks neuroplasticity and language development in children from disadvantaged backgrounds». Frontiers in Psychology, 5(DEC), 1-9.

LEONARD, LAURENCE B. (1998). Children with Specific Language Impairments. Cambridge, MA: MIT Press.

LiNDSAY, GEOFF; DockRell, Julie E.; MACKIE, ClaRe (2008). «Vulnerability to bullying in children with a history of specific speech and language difficulties». European Journal of Special Needs Education, 23(1), 1-16. 
LindSAY, GeOFF; Dockrell, Julie E.; Strand, SteVe (2007). «Longitudinal patterns of behaviour problems in children with specific speech and language difficulties: Child and contextual factors». British Journal of Educational Psychology, 77(4), 811-828.

Lutchmaya, Svetlana; Baron-Cohen, Simon; Raggatt, Peter (2002). «Foetal testosterone and eye contact in 12-month-old human infants». Infant Behavior and Development, 25(3), 327-335.

Maggiolo, Mariangela; Varela, Virginia; Arancibia, Claudia; Ruiz, Felipe (2014). «Dificultades de lenguaje en niños preescolares con antecedente de prematuridad extrema». Revista Chilena de Pediatría, 85(3), 319-327.

Maria-Mengel, Margaret Rose Santa; Linhares, Maria Beatriz Martins (2007). «Risk factors for infant developmental problems». Revista Latino-Americana de Enfermagem, 15(spe), 837-842.

Marton, Klara; Abramoff, Brocha; RosenzWeig, Shari (2005). «Social cognition and language in children with specific language impairment (SLI)». Journal of Communication Disorders, 38(2), 143-162.

McCABE, PAUL C. (2005). «Social and Behavioral Correlates of Preschoolers». Psychology, 42(4).

McKean, Cristina; Wraith, Darren; Eadie, Patricia; Cook, Fallon; Mensah, Fiona; REILLY, SHEENA (2017). «Subgroups in language trajectories from 4 to 11 years: the nature and predictors of stable, improving and decreasing language trajectory groups». Journal of Child Psychology and Psychiatry, 10, 1081-1091.

Norbury, Courtenay Frazier; Gooch, Debbie; Wray, Charlotte; Baird, Gillian; Charman, Tony; Simonoff, Emily; Vamvakas, George; Pickles, Andrew (2016). «The impact of nonverbal ability on prevalence and clinical presentation of language disorder: evidence from a population study». Journal of Child Psychology and Psychiatry, 57(11), 1247-1257.

Özcebe, Esra; Noyan Erbas, Aysin; Karahan TiĞRAK, TuĞÇE (2019). «Analysis of behavioural characteristics of children with developmental language disorders». International Journal of Speech-Language Pathology, 1-7.

Rakhlin, Natalia; Kornilov, Sergey A.; Palejev, Dean; Koposov, Roman A.; Chang, JOSEPH T.; GRIGORENKO, ELENA L. (2013). «The language phenotype of a small geographically isolated Russian-speaking population: Implications for genetic and clinical studies of developmental language disorder». Applied Psycholinguistics, 34(5), 971-1003.

REDMOnd, SEAn M. (2011). «Peer victimization among students with specific language impairment, attention-deficit/hyperactivity disorder, and typical development». Language, Speech, and Hearing Services in Schools, 42(October), 520-536.

Reilly, Sheena; Wake, Melissa; Ukoumunne, Obioha C.; Bavin, Edith; Prior, Margot; Cini, EileEn; Conway, Laura; Eadie, Patricia; Bretherton, LeSley (2010). «Predicting language outcomes at 4 years of age: findings from Early Language in Victoria Study.». Pediatrics, 126(6), e1530-7.

Romeo, Rachel R.; Leonard, Julia A.; Robinson, Sydney T.; West, Martin R.; Mackey, Allyson P.; Rowe, Meredith L.; Gabrieli, John D. E. (2018). «Beyond the 30-MillionWord Gap: Children's Conversational Exposure Is Associated With Language-Related Brain Function». Psychological Science, 095679761774272.

Samson, Andrea C.; Van Den Bedem, Neeltje P.; Dukes, Daniel; Rieffe, Carolien (2020). «Positive aspects of emotional competence in preventing internalizing symptoms in children with and without developmental language disorder: A longitudinal approach». Journal of Autism and Developmental Disorders, (0123456789). 
SERna, Cristina; MartíneZ, IsABEL (2019). «Parental Involvement as a Protective Factor in School Adjustment among Retained and Promoted Secondary Students». Sustainability, 11(24), 7080 .

SHOGREN, KARRIE A.; LUCKASSON, RUTH; SCHALOCK, ROBERT L. (2014). «The definition of "Context" and its application in the field of intellectual disability». Journal of Policy and Practice in Intellectual Disabilities, 11(2), 109-116.

Smith, Jodie; LeVickis, Penny; Eadie, Tricia; Bretherton, Lesley; Conway, laura; GOLDFELD, SHARON (2019). «Associations between early maternal behaviours and child language at 36 months in a cohort experiencing adversity». International Journal of Language and Communication Disorders, 54(1), 110-122.

Smith, Peter K.; López-Castro, Leticia; Robinson, Susanne; Görzig, Anke (2019). «Consistency of gender differences in bullying in cross-cultural surveys». Aggression and Violent Behavior, 45, 33-40.

Spaulding, Tammie J.; Plante, Elena; Vance, Rebecca (2008). «Sustained selective attention skills of preschool children with specific language impairment: Evidence for separate attentional capacities». Journal of Speech, Language and Hearing Research, 51(1), 16-34.

St Clair, Michelle C.; Forrest, Claire L.; Yew, Shaun Goh KoK; Gibson, Jenny L. (2019). «Early Risk Factors and Emotional Difficulties in Children at Risk of Developmental Language Disorder: A Population Cohort Study». Journal of Speech, Language, and Hearing Research, 62(8), 2750-2771.

St Clair, Michelle C..; Pickles, Andrew; Durkin, Kevin; Conti-Ramsden, Gina (2011). «A longitudinal study of behavioral, emotional and social difficulties in individuals with a history of specific language impairment (SLI)». Journal of Communication Disorders, 44(2), 186-199.

Stark, Rachel E.; Tallal, Paula (1981). «Selection of children with specific language deficits». Journal of Speech and Hearing Disorders, 46(2), 114-122.

Sureda García, Inmaculada; Valera-Pozo, Mario; Aguilar-Mediavilla, Eva (2019). «Afectación del lenguaje debido a variables sociales y emocionales». A Dificultades del lenguaje en los trastornos del desarrollo Vol III: factores de riesgo y dificultades comórbidas (p. 71-23). Barcelona: Editorial UOC.

Tamis-Lemonda, Catherine S.; Song, Lulu; Leavell, Ashley Smith; Kahana-Kalman, RONIT; YOSHIKAWA, HIROZAKU (2012). «Ethnic differences in mother-infant language and gestural communications are associated with specific skills in infants». Developmental Science, 15(3), 384-397.

Tomblin, J. Bruce; Records, Nancy L.; Buckwalter, Paula; Zhang, Xuyang; Smith, ELAINE; O'BRIEN, MARLEA (1997). «Prevalence of Specific Language Impairment in kindergarten children». Journal of Speech, Language, and Hearing Research, 40(December 1997), 1245-1260.

Toseeb, Umar; Oginni, Olakunle; Dale, Philip (2020). «Developmental Language Disorder and Psychopathology: Disentangling Shared Genetic and Environmental Influences». Pre print, 1-34.

Toseeb, UMar; St Clair, Michelle C. (2020). «Trajectories of prosociality from early to middle childhood in children at risk of Developmental Language Disorder». Journal of Communication Disorders, 85(February 2019), 105984.

TOUGH, JOAN (1987). El lenguaje oral en la escuela. Una guía de observación y actuación para el maestro. Madrid: Visor. 
Valera-Pozo, Mario; Adrover-Roig, Daniel; Pérez-Castelló, Josep A.; SanchezAZANZA, Victor A.; AgUilar-MEdiavilla, Eva (2020). «Behavioral, emotional and school adjustment in adolescents with and without developmental language disorder (DLD) is related to family involvement». International Journal of Environmental Research and Public Health, 17(6), 1949.

Van Den Bedem, Neeltje P.; Dockrell, Julie E.; Alphen, Petra M.; Rooij, Mark; SAMSON, ANDREA C.; HARJunEN, Elina L.; RiEFFE, CAROLIEN (2018). «Depressive symptoms and emotion regulation strategies in children with and without developmental language disorder: a longitudinal study». International Journal of Language \& Communication Disorders, 53(6), 1110-1123.

Voci, Sabrina C.; Beitchman, Joseph H.; Brownlie, Elizabeth; Wilson, Beth (2006). «Social anxiety in late adolescence: The importance of early childhood language impairment». Journal of Anxiety Disorders, 20(7), 915-930.

Wadman, Ruth; Durkin, Kevin; Conti-Ramsden, Gina (2011). «Social stress in young people with specific language impairment.». Journal of adolescence, 34(3), 421-31.

Whitehouse, ANDrew Jo (2010). «Is There a Sex Ratio Difference in the Familial Aggregation of Specific Language Impairment? A Meta-Analysis». Journal of Speech, Language, and Hearing Research, 53(4), 1015-1025.

Yew, SHAUn GoH KoK; O’KeARNEY, RichaRd (2013). «Emotional and behavioural outcomes later in childhood and adolescence for children with specific language impairments: metaanalyses of controlled prospective studies». Journal of Child Psychology and Psychiatry, 54(5), 516-524.

Yew, SHAun GOH KoK; O'KeARneY, RichaRD (2015). «Early language impairments and developmental pathways of emotional problems across childhood». International Journal of Language \& Communication Disorders, 50(3), 358-373.

Zambrana, Imac Maria; Pons, Francisco; Eadie, Patricia; Ystrom, Eivind (2014). «Trajectories of language delay from age 3 to 5: Persistence, recovery and late onset». International Journal of Language and Communication Disorders, 49(3), 304-316.

Zubrick, StePhen R.; TAYlor, CATHERINe L.; Christensen, DANiEl (2015). «Patterns and predictors of language and literacy abilities 4-10 years in the longitudinal study of Australian children». PLoS ONE, 10(9), 1-29. 